\title{
A new roadmap for the breeding of disease-resistant and high-yield crops
}

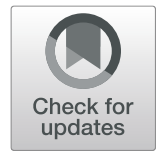

Yiming Wang ${ }^{1,2^{*}}$ and Suomeng Dong ${ }^{1,2^{*}}$ (D)

\begin{abstract}
Breeding of disease-resistant and high-yield crops is essential to meet the increasing food demand of the global population. However, the breeding of such crops remains a significant challenge for scientists and breeders. Two recent discoveries may help to overcome this challenge: the discovery of a novel molecular framework to fine-tune disease resistance and yields that includes epigenetic regulation of antagonistic immune receptors, and the discovery of a $\mathrm{Ca}^{2+}$ sensor-mediated immune repression network that enables the transfer of subspecies-specific and broad-spectrum disease resistance. These breakthroughs provide a promising roadmap for the future breeding of disease resistant crops.
\end{abstract}

Keywords: Rice, Immunity, $\mathrm{Ca}^{2+}$ signaling, Reactive oxygen species; ROD1; Pigm

\section{Introduction}

Global food production needs to increase by at least $60 \%$ to meet the food demand of the estimated global population of 10 billion people in 2050 (Food and Agriculture Organization, 2019). To meet this goal, a significant increase of crop production is needed. It is estimated that the global crop loss of $17-30 \%$, depending on different crops, is due to plant pathogens and pests (Savary et al. 2019). However, improving crop resistance to pathogens and pests is challenging, since host resistance is frequently accompanied by growth disadvantage and yield penalty, which is known as the defense-growth tradeoff (Yang et al., 2012). To address this challenge, extensive research efforts have been aimed at generating pathogen-resistant crops with less yield penalty.

Zuhua He's group, from the CAS Center for Excellence in Molecular Plant Sciences, study the mechanistic interaction between resistance and yield and aim to find a balanced solution for crop breeding. Gumei4, a highly resistant rice variety containing the Pigm resistance locus, has been used to improve the resistance of rice against the rice blast fungus for over 50 years. However,

\footnotetext{
* Correspondence: ymwang@njau.edu.cn; smdong@njau.edu.cn

${ }^{1}$ Key Laboratory of Biological Interaction and Crop Health, Department of Plant Pathology, Nanjing Agricultural University, Nanjing 210095, China Full list of author information is available at the end of the article
}

the molecular mechanism of Pigm-mediated resistance has remained unclear until recently. Deng et al. developed near-isogenic rice lines containing the Pigm resistance locus (NIL-Pigm) (Deng et al., 2006). By map-based cloning and gamma-ray-mutagenized Gumei4 populations, the Pigm locus was functionally identified; it contains three intact nucleotide-binding leucine-rich repeats (NLR) receptors, 10 potential pseudogenes, and one retrotransposon. The NLR receptor Pigm Resistant (PigmR) was further identified as a broad resistance gene against the rice blast fungus. Transgenic rice plants expressing PigmR significantly increased their resistance against the rice blast fungus but also showed yield penalty. However, Deng et al. also noticed that no significant yield penalty was observed in NIL of Pigm in either indica or japonica rice varieties, suggesting that a defense-growth balance was maintained by genes in the Pigm locus.

The ectopic expression of another NLR receptor in the Pigm locus, Pigm Susceptible (PigmS), reduces PigmRmediated resistance by forming PigmR-PigmS heterodimers. This reduction of host immunity in PigmS-expressing plants is accompanied by an increase in grain yield due to an increase in seed settings. However, the mechanism of the PigmR-PigmS-mediated defensegrowth tradeoff inside the host is still unclear. Deng 
et al. further noticed that the expression of PigmS under native promoters did not affect disease resistance mediated by PigmR, suggesting that the expression pattern may be essential for the function of PigmS.

RNA-directed DNA methylation ( $\mathrm{RdDM})$ is required for the epigenetic silence of plant transposable elements (Cui and Cao, 2014). The lower expression of PigmS in leaves was affected by two tandem miniature transposons (MITE1 and MITE2) in its promoter region (Deng et al., 2017). Accumulation of 24nucleotide-small interfering RNA is responsible for the RdDM-mediated silencing of PigmS at MITE1 and MITE2 regions in leaves. Therefore, under nondisease conditions, the total yield balance was mediated by PigmR-decreased grain weight and PigmS-enhanced seed setting. These findings demonstrated that epigenetic regulation of the NLR-repressive pair gene balances the defense-growth tradeoff in rice.

NLR genes are highly polymorphic in rice germplasm and typically confer specific resistances. Is it possible to modulate conserved genes to enhance the broad resistance of plants against multiple pathogens? Recently, Zuhua He's group identified a broad-spectrum resistant rice variant, Resistance of rice to disease1 (rod1), through large-scale screening of germplasm and breeding collections. The rod 1 mutant exhibits high resistance against the rice blast fungus, rice bacterial blight, and rice sheath blight, which are the major threats to rice productivity (Gao et al. 2021). Map-based cloning showed that ROD1 encodes a $\mathrm{C} 2$-domain $\mathrm{Ca}^{2+}$ sensor protein exhibiting $\mathrm{Ca}^{2+}$ binding activity. Loss of $\mathrm{Ca}^{2+}$ binding disables the lipid binding and plasma membrane localization of ROD1, causing failure to rescue growth retardation and immune activation in rod1 (Gao et al. 2021). These findings illustrate that the $\mathrm{Ca}^{2+}$ binding activity is crucial for the function of ROD1.

Interestingly, the rod1 mutant exhibits high accumulation and induction levels of reactive oxygen species (ROS) in rice, highlighting a potential link between $\mathrm{Ca}^{2+}$ and ROS signaling. The ROD1interacting protein CatB, a ROS-scavenging enzyme, was detected through a yeast-two-hybrid screening system. These findings illustrate the possible role of ROD1 in ROS modulation through CatB. ROD1 recruits the localization of $\mathrm{CatB}$ from the peroxisome to the plasma membrane and promotes the ROSscavenging activity of CatB (Gao et al. 2021). Moreover, co-expression of ROD1-CatB significantly reduces coiled-coil NLR-mediated cell death and ROS accumulation in tobacco leaves. These findings illustrate that the ROD1-CatB interaction is required to suppress rice immunity through modulating ROS accumulation. Consistent with the defense-growth tradeoff hypothesis, the rod1 mutant exhibits a phenotype of compromised plant growth and reduced productivity. This raises the question of whether it is possible to enhance pathogen resistance without yield penalty. To answer this question, Gao et al. identified the natural variations of ROD1 from 262 cultivated Asian rice accessions. Interestingly, they found a nonsynonymous nucleotide polymorphism, SNP1 ${ }^{\mathrm{A} / \mathrm{C}}$ (an amino acid change from proline to threonine), which contributes to subspecies-specific disease resistance. The ROD1 $\mathrm{SNP}^{\mathrm{A}}$ is mainly retained in indica rice varieties, whereas $\mathrm{SNP1}{ }^{\mathrm{C}}$ is predominant in both wild rice and japonica rice varieties. A complementation assay using ROD1 $\mathrm{SNP}^{\mathrm{A}}$ and $\mathrm{SNP} 1^{\mathrm{C}}$ was carried out in the rod1 mutant and showed that ROD1 $\mathrm{SNP} 1^{\mathrm{C}}$ affected neither pathogen resistance nor plant growth. However, ROD1 SNP1 ${ }^{\mathrm{A}}$, which showed a reduction of $\mathrm{H}_{2} \mathrm{O}_{2}$ hydrolysis activity, transferred the enhanced disease resistance without growth repression. This result is consistent with evolutionary analysis showing that the ROD1 in indica rice undergoes differential selection during rice adaptation.

The identification of PigmR and ROD1, which either confers broad-spectrum blast resistance or suppresses immunity, was part of a large-scale study screening highly resistant plants in the field. PigmR exhibits genus-specific resistance against the rice blast fungus, whereas rod1 exhibits broad resistance against multiple pathogens. Although these two genes provide different molecular mechanisms, their good performances in terms of pathogen resistance under natural conditions make them promising candidates for utilizing those resistances in future breeding processes. The maize ZmROD1 mutant also exhibits enhanced resistance against the pathogenic fungus $R$. solani, suggesting that ROD1 is functionally conserved across cereal plants (Gao et al. 2021). Moreover, pathogenderived selection pressure during long-term coevolution resulted in the maintaining of beneficial gene variations in plants. A single SNP in ROD1 does not cause differences in growth but does improve field disease resistance. These findings provide a roadmap for the breeding of highly resistant crops with less yield penalty. Utilizing the available information on natural variation may provide a deeper understanding of molecular and evolutionary mechanisms in plant immunity.

Information on the crosstalk between different signaling pathways in plants remains incomplete. $\mathrm{Ca}^{2+}$ and ROS are required for multiple processes in plants, including development and biotic and abiotic responses. ROD1 bridges $\mathrm{Ca}^{2+}$ signaling to ROS signaling in rice defenses, and this mechanism is likely to occur in other plants. A better understanding of signaling crosstalk, especially the identification of joint points of signaling 
networks, can lead to a better understanding of tradeoff between defense and growth, biotic and abiotic responses, and other mechanisms. Identifying these antagonistic biological processes provides a promising outlook for the future breeding of resistant crops.

\section{Abbreviations}

CatB: Catalase B; NIL: Near-isogenic rice lines; NLR: nucleotide-binding leucine-rich repeats; PigmR: Pigm Resistant; PigmS: Pigm Susceptible; RdDM: RNA-directed DNA methylation; ROD1: RESISTANCE OF RICE TO DISEASE1; ROS: Reactive oxygen species; SNP: Synonymous nucleotide polymorphism

\section{Acknowledgements}

We thank Weibing Yang for critical reading of the manuscript.

\section{Authors' contributions}

Y.W. and S.D. conceived the project and wrote the paper. Both author(s) read and approved the final manuscript.

\section{Funding}

Y.W. was supported by the grants from the National Natural Science Foundation of China (32172420), and the Fundamental Research Funds for the Central Universities (KYXK202009; ZJ21195012). S.D. received support from National Natural Science Foundation of China (31721004).

\section{Availability of data and materials}

Not applicable.

\section{Declarations}

\section{Competing interests}

The authors declare that they have no competing interests.

\section{Author details}

'Key Laboratory of Biological Interaction and Crop Health, Department of Plant Pathology, Nanjing Agricultural University, Nanjing 210095, China. ${ }^{2}$ Key Laboratory of Integrated Management of Crop Disease and Pests, Ministry of Education, Nanjing Agricultural University, Nanjing, China.

Received: 19 October 2021 Accepted: 6 December 2021

Published online: 29 December 2021

\section{References}

Cui X, Cao X (2014) Epigenetic regulation and functional exaptation of transposable elements in higher plants. Curr Opin Plant Biol 21:83-88. https://doi.org/10.1016/j.pbi.2014.07.001

Deng Y, Zhai K, Xie Z, Yang D, Zhu X, Liu J, Wang X, Qin P, Yang Y, Zhang G, Li Q, ZHang J, Wu S, Milazzo J, Mao B, Wang E, Xie H, Tharreu D, He Z (2017) Epigenetic regulation of antagonistic receptors confers rice blast resistance with yield balance. Science 355(6328):962-965. https://doi.org/10.1126/ science.aai8898

Deng Y, Zhu X, Shen Y, He Z (2006) Genetic characterization and fine mapping of the blast resistance locus Pigm(t) tightly linked to Pi2 and Pi9 in a broadspectrum resistant Chinese variety. Theor Appl Genet 113(4):705-713. https:// doi.org/10.1007/s00122-006-0338-7

Food and Agriculture Organization (2019) The State of Food and Agriculture 2019: Moving Forward on Food Loss and Waste Reduction. http://www.fao. org/state-of-food-agriculture/2019/en. Accessed 16 October 2019

Gao M, He Y, Yin X, Zhong X, Yan B, Wu Y, Chen J, Li X, Zhai K, Huang Y, Gong X, Chang H, Xie S, Liu J, Yue J, Xu J, Zhang G, Deng Y, Wang E, Tharreau D, Wang G, Yang W, He Z (2021) $\mathrm{Ca}^{2+}$ sensor-mediated ROS scavenging suppresses rice immunity and is exploited by a fungal effector. Cell 184(21): 1-14. https://doi.org/10.1016/j.cell.2021.09.009

Joo J, Lee YH, Song SI (2014) Rice CatA, CatB, and CatC are involved in environmental stress response, root growth, and photorespiration, respectively. J Plant Biol 57(6):375-382. https://doi.org/10.1007/s12374-014-03 83-8
Savary S, Willocquet L, Pethybridge SJ, Esker P, McRoberts N, Nelson A (2019) The global burden of pathogens and pests on major food crops. Nat Ecol Evol 3(3):430-439. https://doi.org/10.1038/s41559-018-0793-y

Yang D, Yao J, Mei C, Tong X, Zeng L, Li Q, Xiao L, Sun T, Li J, Deng X, Lee CM, Thomashow MF, Yang Y, He Z, He SY (2012) Plant hormone jasmonate prioritizes defense over growth by interfering with gibberellin signaling cascade. PNAS 109(19):E1192-E1200. https://doi.org/10.1073/ pnas.1201616109

\section{Publisher's Note}

Springer Nature remains neutral with regard to jurisdictional claims in published maps and institutional affiliations. 